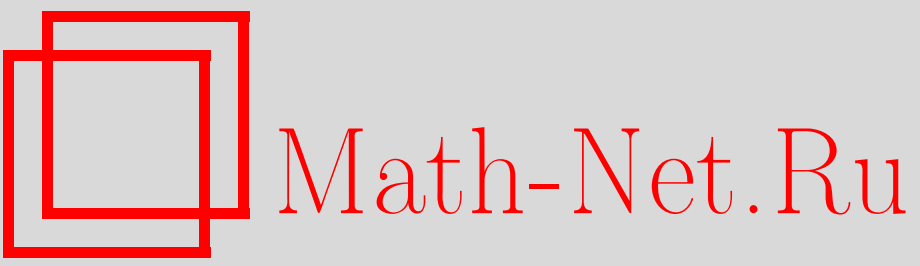

В. М. Ульянов, В. А. Хрипин, А. Ю. Кирьянов, Колебательное движение тела с изменяющимся центром масс на гибких подвесах, Итоги науки и техн. Сер. Соврем. мат. и ее прил. Темат. обз., 2020, том 186, 126-137

DOI: https://doi.org/10.36535/0233-6723-2020-186-126-137

Использование Общероссийского математического портала Math-Net.Ru подразумевает, что вы прочитали и согласны с пользовательским соглашением

http://www.mathnet.ru/rus/agreement

Параметры загрузки:

IP: 54.237 .206 .68

26 апреля 2023 г., 10:30:43 


\title{
КОЛЕБАТЕЛЬНОЕ ДВИЖЕНИЕ ТЕЛА \\ С ИЗМЕНЯЮЩИМСЯ ЦЕНТРОМ МАСС \\ НА ГИБКИХ ПОДВЕСАХ
}

\author{
(c) 2020 г. $\quad$ В. М. Ульянов, В. А. ХРИпИн, А. Ю. КИРЬЯноВ
}

\begin{abstract}
АннотАция. Получено нелинейное дифференциальное уравнение второго порядка, описывающее вынужденные колебания в доильном аппарате. Приведены приближенные аналитические решения полученных дифференциальных уравнений.
\end{abstract}

Ключевые слова: дифференциальное уравнение, колебания, амплитуда, угол отклонения, гибкий подвес, центр масс, кинетический момент.

\section{OSCILLATORY MOTION OF A BODY WITH A CHANGING CENTER OF MASS ON FLEXIBLE SUSPENSIONS}

\author{
(c) 2020 V. M. ULYANOV, V. A. KHRIPIN, A. YU. KIRYANOV
}

\begin{abstract}
We obtain a nonlinear second-order differential equation that describes forced vibrations in a milking machine. Approximate analytical solutions of the obtained differential equations are given.
\end{abstract}

Keywords and phrases: differential equation, oscillations, amplitude, deflection angle, flexible suspension, center of mass, angular momentum.

AMS Subject Classification: $34 \mathrm{~K} 06$

1. Введение. Результаты данных исследований носят прикладной характер. При доении коров используется физиологически адаптированный доильный аппарат, коллектор которого снабжен подвижным телом, изменяющим центр масс подвесной части доильного аппарата в зависимости от такта работы пульсатора. Это позволяет обеспечить стабильность процесса выведения молока (см. $[6-8])$.

2. Основные результаты. Расчетная схема взаимодействия системы представлена на рис. 1. Введем систему координат, за начало отсчета примем точку $O_{1}$, ось $O_{1} X$ справа налево, а $O_{1} Y-$ вниз. Примем обозначения: $m$ - масса поршня, кг; $M$ - масса направляющей (цилиндра) с центром тяжести в точке $O ; S$-расстояние от правого конца цилиндра коллектора до поршня, м; $\phi-$ угол отклонения подвеса (молочного патрубка стакана) от вертикального положения, рад; $a$, $l$ - длина соответственно направляющей и подвеса, м; $T_{1}, T_{2}$ - реакции со стороны направляющей с поршнем на соски соответственно правой и левой долей вымени. Значение реакций $T_{1}, T_{2}$, таковы, что стаканы будут неподвижны на сосках вымени, т.е. длины подвесов $O_{1} A=O_{2} B=l=$ const 


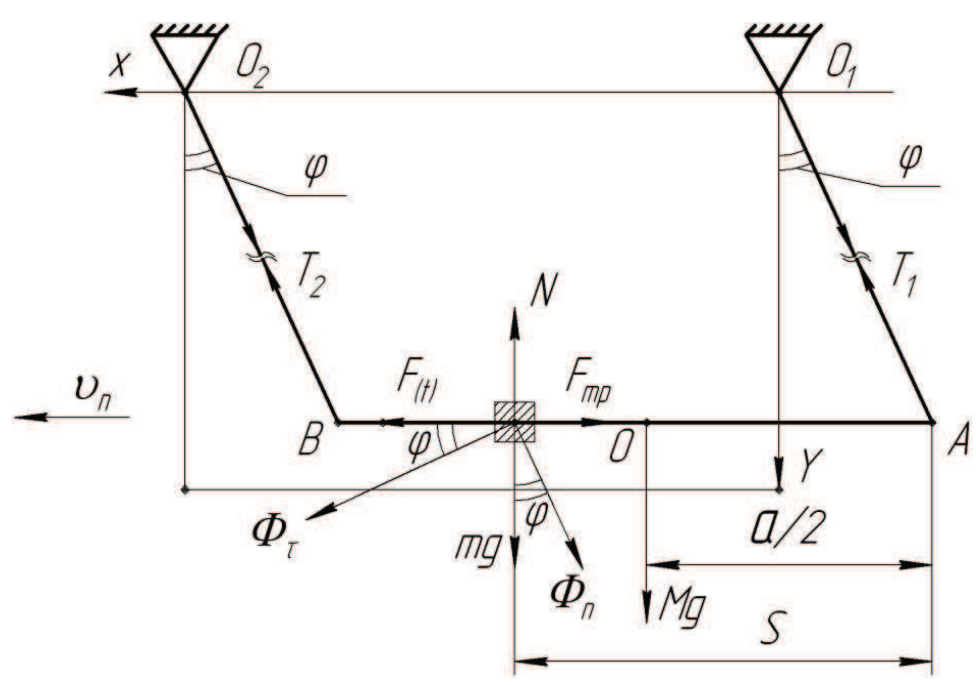

Рис. 1. Схема траектории движения тела

при движении всей системы. Выберем произвольное положение системы и приложим действующие силы (рис. 1).

Взаимодействие подвесной части доильного аппарата с выменем коровы удобно рассматривать по этапам. При подключении аппарата к вакууму перепад давлений передается на поршень, который приходит в относительное движение по направляющей. Направляющая, вдоль которой перемещается поршень, совершает поступательное движение, т.е. скорости всех ее точек одинаковы как по направлению, так и по модулю. Точки $A$ и $B$, в которых направляющая соединена с тягами подвеса, совершают вращательное движение при изменении угла поворота $\phi$. В то же время точки $A$ и $B$ являются точками направляющей $A B$. Значит, скорости поступательного движения остальных точек направляющей по отношению к неподвижным токам подвеса $O_{1}, O_{2}$ будут направлены по касательной к окружностям движения точек $A$ и $B$, т. е. перпендикулярно тягам подвесов $O_{1} B$ и $O_{2} A$. Направляющая $A B$ при колебаниях тяг подвесов будет занимать всегда горизонтальное положение. Уравнение относительного движения поршня будет

$$
m \ddot{S}=F_{(t)}+\Phi+F_{m}
$$

где $F_{(t)}$ - сила от перепада давлений, действующая на поршень, Н; $\Phi$ - сила инерции поршня, Н; $F_{m}$ - сила трения, Н. Проекция на направляющую $A B$ силы инерции $\Phi$ : нормальная составляющая

касательная составляющая

$$
\Phi_{n}=m l(\dot{\phi})^{2},
$$

$$
\Phi_{\tau}=m l \ddot{\phi} .
$$

Сила трения по закону сухого трения Кулона

$$
F_{m}=f \cdot N
$$

где $f$-коэффициент трения; $N$ - сила нормального давления, Н. Сила нормального давления (рис. 1) составит:

$$
N=m g+\Phi_{n} \cdot \cos \phi+\Phi_{\tau} \cdot \sin \phi .
$$

Тогда формула (1) с учетом выражений со (2) по (5) примет вид:

$$
m \ddot{S}=F_{(t)}+\left[m l \ddot{\phi} \cdot \cos \phi-m l(\dot{\phi})^{2} \cdot \sin \phi\right]-f\left[m g+m l(\dot{\phi})^{2} \cdot \cos \phi+m l \ddot{\phi} \cdot \sin \phi\right]
$$




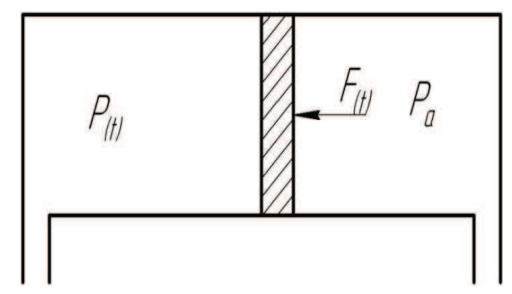

Рис. 2. Схема к расчету силы, действующей на поршень

При работе на поршень действует сила от перепада давлений. При откачивании воздуха из герметичной камеры скорость откачивания замедляется по мере уменьшения в ней давления (см. [5]). По аналогии будем считать, что скорость пропорциональна оставшейся разности давлений в камере. Пусть $t_{0}$ - время начала откачивания воздуха, $\mathrm{c} ; t$-время, прошедшее от начала откачивания, с; $P_{a}, P_{(t)}$ - соответственно атмосферное и текущее давления в камерах цилиндра, $\mathrm{H} / \mathrm{м}^{2}$; $P_{h}$ - рабочее давление в вакуумпроводе, $\mathrm{H} / \mathrm{M}^{2}$. Согласно предположению, что скорость изменения давления $\frac{d P}{d t}$ пропорциональна оставшейся разности давлений $\left(P_{h}-P_{(t)}\right)$, запишем

$$
\frac{d P}{d t}=k\left(P_{h}-P_{(t)}\right) \text { или } \frac{d P}{P_{(t)}-P_{h}}=-k d t,
$$

где $k$ - коэффициент пропорциональности, $k>0$. Интегрируя выражение $(7)$, получаем

$$
\ln \left|P_{(t)}-P_{h}\right|=-k\left(t-t_{0}\right)+C_{1} .
$$

Снимая знак модуля за счет значения новой постоянной $C$, запишем

$$
P_{(t)}-P_{h}=C \cdot e^{-k\left(t-t_{0}\right)} \text { или } P_{(t)}=P_{h}+C \cdot e^{-k\left(t-t_{0}\right)} .
$$

Считая, что в начале процесса откачивания давление в камере цилиндра коллектора совпадает с атмосферным, получаем начальные условия к уравнению $\left.(8) P_{(t)}\right|_{t=t_{0}}=P_{a}$. Решая выражение (8) относительно постоянной $C$ с учетом начальных условий, имеем $C=P_{a}-P_{h}$. Подставляя значение константы $C$ в формулу (8), получаем закон изменения давления в камере коллектора при откачивании из нее воздуха.

$$
P_{(t)}=P_{h}+\left(P_{a}-P_{h}\right) e^{-k\left(t-t_{0}\right)}
$$

Коэффициент пропорциональности $k$ имеет размерность $c^{-1}$. Он учитывает как объем откачиваемого воздуха из камеры, так и возникающее при этом сопротивление движению воздуха. Значение коэффициента можно определить аналитически, а затем ввести уточнение при эксперименте. Так, сравнивая зависимость (8) с формулами, приведенными Г. Д. Талалаевым (см. [4]) для откачивания воздуха из управляющей камеры пульсатора, можно сделать вывод, что коэффициент $k$ при $t=0$ определяется зависимостью:

$$
k=\frac{\pi \cdot d_{m}^{4} P_{h}}{c \cdot 128 V \cdot l_{m} \cdot \eta}
$$

где $c$-опытный коэффициент, предварительно $c=1 ; V$-объем камеры цилиндра, из которой откачивается воздух, $\mathrm{m}^{3} ; l_{m}, d_{m}$ - соответственно длина и диаметр трубки, соединяющей пульсатор с камерой цилиндра коллектора, м; $\eta$ - динамическая вязкость, Па · . Для текущего давления $P(t)$ в камере цилиндра выполняется неравенство $P_{h} \leqslant P(t) \leqslant P_{a}$. Принимаем, что с одной стороны на поршень действует атмосферное давление $P_{a}$, а с противоположной - давление $P_{(t)}$ (рис. 2).

Пусть $S_{1}$ - площадь поршня. Считая, что отсутствует перетекание воздуха из камер цилиндра, получим силу, движущую поршень в виде:

$$
F(t)=\left(P_{a}-P_{(t)}\right) S_{1}=\left(P_{a}-P_{h}-\left(P_{a}-P_{h}\right) \cdot e^{-k\left(t-t_{0}\right)}\right) S_{1}=P_{b}\left(1-e^{-k\left(t-t_{0}\right)}\right) S_{1},
$$




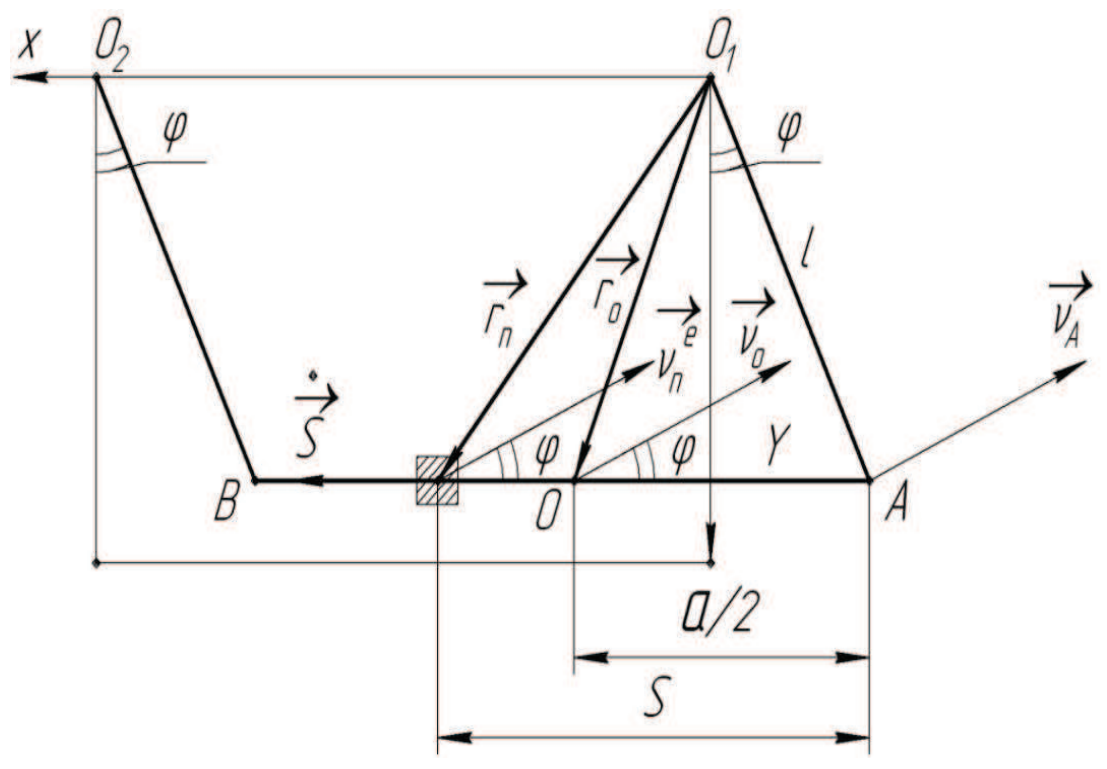

Рис. 3. Схема к определению движения системы

где $P_{b}=P_{a}-P_{h}-$ величина вакуума в цилиндре, $\mathrm{H} / \mathrm{м}^{2}$. Окончательно уравнение относительного движения поршня (6) с учетом формулы (11) будет

$$
m \ddot{S}=P_{b} \cdot S_{1}\left(1-e^{-k\left(t-t_{0}\right)}\right)-f \cdot m g+m l \ddot{\phi}(\cos \phi-f \sin \phi)-m l(\dot{\phi})^{2}(\sin \phi+f \cdot \cos \phi) .
$$

Чтобы определить движение системы в целом, воспользуемся теоремами о движении центра масс и об изменении кинетического момента относительно точки $O_{1}$. Последняя теорема запишется следующим образом (см. [3]):

$$
\frac{d K_{O_{1}}}{d t}=\sum M_{O_{1}}\left(\boldsymbol{F}_{\boldsymbol{i}}\right)
$$

На рис. 3 представлена схема к определению движения системы.

Кинетический момент системы относительно точки $O_{1}$ в векторной форме:

$$
\boldsymbol{K}_{O_{1}}=\boldsymbol{r}_{n} \cdot m \boldsymbol{\vartheta}_{n}+\boldsymbol{r}_{0} \cdot M \boldsymbol{\vartheta}_{0},
$$

где $\boldsymbol{r}_{n}$ и $\boldsymbol{r}_{0}$-соответственно радиус-вектор поршня и радиус-вектор центра масс (точка $O$ ), м; $\boldsymbol{\vartheta}_{n}$ и $\boldsymbol{\vartheta}_{0}$ - вектор скорости поршня и центра масс направляющей, м/с; $m, M$ - соответственно массы поршня и направляющей, кг. Радиус-вектор поршня можно записать следующим образом в матричном виде:

$$
\boldsymbol{r}_{n}=\left(\begin{array}{c}
r_{n x} \\
r_{n y} \\
r_{n z}
\end{array}\right)=\left(\begin{array}{c}
S-l \sin \phi \\
l \cos \phi \\
0
\end{array}\right)
$$

где $r_{n x}, r_{n y}, r_{n z}$ - проекции радиус-вектора на оси, равные $r_{n x}=S-l \sin \phi ; r_{n y}=l \cos \phi ; r_{n z}=0$. Абсолютная скорость поршня будет:

$$
\boldsymbol{\vartheta}_{n}=\boldsymbol{\vartheta}_{n}^{4}+\boldsymbol{\vartheta}_{a}^{4}
$$

где $\boldsymbol{\vartheta}_{n}^{4}, \boldsymbol{\vartheta}_{a}^{4}-$ соответственно переносная и относительная скорости поршня, м/с. Переносная и относительная скорости поршня соответственно равны

$$
\boldsymbol{\vartheta}_{n}^{e}=\left(\begin{array}{c}
-l \dot{\phi} \cos \phi \\
-l \dot{\phi} \sin \phi \\
0
\end{array}\right) ; \quad \boldsymbol{\vartheta}_{n}^{a}=\dot{\boldsymbol{S}}=\left(\begin{array}{c}
\dot{S} \\
0 \\
0
\end{array}\right)
$$


Тогда абсолютная скорость поршня примет вид

$$
\boldsymbol{\vartheta}_{n}=\left(\begin{array}{c}
\dot{S}-l \dot{\phi} \cos \phi \\
-l \dot{\phi} \sin \phi \\
0
\end{array}\right)
$$

Определим кинетический момент поршня $K_{O_{1(n)}}$ относительно точки $O_{1}$.

$$
\begin{aligned}
& K_{O_{1(n)}}= \boldsymbol{r}_{n} \cdot m \boldsymbol{\vartheta}_{n}=m\left|\begin{array}{ccc}
\boldsymbol{i} & \boldsymbol{j} & \boldsymbol{k} \\
r_{n x} & r_{n y} & r_{n z} \\
\vartheta_{n x} & \vartheta_{n y} & \vartheta_{n z}
\end{array}\right|=m\left|\begin{array}{ccc}
\boldsymbol{i} & \boldsymbol{j} & \boldsymbol{k} \\
S-l \sin \phi & l \cos \phi & 0 \\
\dot{S}-l \dot{\phi} \cos \phi & -l \dot{\phi} \sin \phi & 0
\end{array}\right|= \\
&=m \cdot \boldsymbol{k}[(S-l \sin \phi)-(l \dot{\phi} \sin \phi)-l \cos \phi(\dot{S}-l \dot{\phi} \cos \phi)]= \\
&=-m l \boldsymbol{k}\left(S \dot{\phi} \sin \phi-l \dot{\phi} \sin ^{2} \phi+\dot{S} \cos \phi-l \dot{\phi} \cos ^{2} \phi\right)=m l(l \dot{\phi}-S \sin \phi \cdot \dot{\phi}-\dot{S} \cos \phi) \boldsymbol{k} .
\end{aligned}
$$

Далее аналогично находим радиус-вектор центра масс (точки $O$ ) направляющей:

$$
\boldsymbol{r}_{0}=\left(\begin{array}{c}
r_{0 x} \\
r_{0 x} \\
r_{0 z}
\end{array}\right)
$$

или

$$
\boldsymbol{r}_{0}=\left(\begin{array}{c}
\frac{a}{2}-l \sin \phi \\
l \cos \phi \\
0
\end{array}\right)
$$

где $r_{0 x}, r_{0 y}, r_{0 Z}$ - проекции радиус-вектора точки $O$ на оси координат, равные $r_{0 x}=\frac{a}{2}-l \sin \phi$; $r_{0 y}=l \cos \phi ; r_{0 z}=0$. Абсолютная скорость точки $O$ будет:

$$
\boldsymbol{\vartheta}_{0}=\left(\begin{array}{c}
\vartheta_{0 x} \\
\vartheta_{0 y} \\
\vartheta_{0 z}
\end{array}\right)=\left(\begin{array}{c}
-l \dot{\phi} \cos \phi \\
-l \dot{\phi} \sin \phi \\
0
\end{array}\right)=-l \dot{\phi}\left(\begin{array}{c}
\cos \phi \\
\sin \phi \\
0
\end{array}\right),
$$

где проекции скорости $\vartheta_{0}$ на оси будут $\vartheta_{0 x}=-\vartheta_{0} \cos \phi ; \vartheta_{0 y}=-\vartheta_{0} \sin \phi ; \vartheta_{0 z}=0 ; \vartheta=l \dot{\phi}_{0}$. Кинетический момент центра масс направляющей относительно точки $O_{1}$ будет иметь вид:

$$
\begin{gathered}
K_{O_{1}(0)}=\boldsymbol{r}_{0} \cdot M \boldsymbol{\vartheta}_{0}=M\left|\begin{array}{ccc}
\boldsymbol{i} & \boldsymbol{j} & \boldsymbol{k} \\
r_{0 x} & r_{0 y} & r_{0 z} \\
\vartheta_{0 x} & \vartheta_{0 y} & \vartheta_{0 z}
\end{array}\right|=-M l \dot{\phi}\left|\begin{array}{ccc}
\boldsymbol{i} & \boldsymbol{j} & \boldsymbol{k} \\
\frac{a}{2}-l \sin \phi & l \cos \phi & 0 \\
\cos \phi & \sin \phi & 0
\end{array}\right|= \\
=-M l \dot{\phi} \boldsymbol{k}\left[\left(\frac{a}{2}-l \sin \phi\right) \sin \phi-l \cos ^{2} \phi\right]=M \cdot l \dot{\phi}\left(l-\frac{a}{2} \sin \phi\right) \boldsymbol{k} .
\end{gathered}
$$

Кинетический момент всей системы относительно точки $O_{1}$ :

$$
\begin{aligned}
K_{O_{1}}=K_{O_{1}(n)}+K_{O_{1}(0)}=m l(l \dot{\phi}- & S \sin \phi \cdot \dot{\phi}-\dot{S} \cos \phi) \boldsymbol{k}+M \cdot l \dot{\phi}\left(l-\frac{a}{2} \sin \phi\right) \boldsymbol{k}= \\
= & {\left[m(l \dot{\phi}-S \sin \phi \cdot \dot{\phi}-\dot{S} \cos \phi)+M\left(l-\frac{a}{2} \sin \phi\right) \dot{\phi}\right] l \cdot \boldsymbol{k} . }
\end{aligned}
$$

Дифференцируем выражение (21), получаем:

$$
\frac{d \boldsymbol{K}}{d t}=\left\{m\left[(l-S \sin \phi) \ddot{\phi}-\ddot{S} \cos \phi-S \cos \phi(\dot{\phi})^{2}\right]+M\left[\left(l-\frac{a}{2} \sin \phi\right) \ddot{\phi}-\frac{a}{2} \cos \phi(\dot{\phi})^{2}\right]\right\} l \cdot \boldsymbol{k} .
$$

Таким образом, определена левая часть формулы (13). Переходим к вычислению ее правой части, то есть к нахождению суммы моментов всех действующих внешних сил относительно точки $O_{1}$. Внешними силами являются сила тяжести поршня $\overrightarrow{m g}$, сила тяжести направляющей системы $\overrightarrow{M g}$ и реакции $\boldsymbol{T}_{1}$ и $\boldsymbol{T}_{2}$. Радиус-вектор поршня

$$
\boldsymbol{r}_{n}=\left(\begin{array}{c}
S-l \sin \phi \\
l \cos \phi \\
0
\end{array}\right)
$$




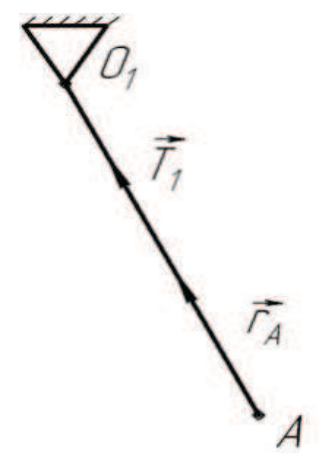

Рис. 4. Схема к расчету момента реакции $T_{1}$

а вектор силы

$$
\overrightarrow{m g}=\left(\begin{array}{c}
0 \\
m g \\
0
\end{array}\right)
$$

тогда момент силы тяжести поршня составит:

$$
M_{O_{1}(\overrightarrow{m g})}=\boldsymbol{r}_{n} \cdot \overrightarrow{m g}=\left|\begin{array}{ccc}
\boldsymbol{i} & \boldsymbol{j} & \boldsymbol{k} \\
S-l \sin \phi & l \cos \phi & 0 \\
0 & m g & 0
\end{array}\right|=m g(S-l \sin \phi) \boldsymbol{k} .
$$

Аналогично радиус-вектор центра тяжести направляющей

$$
\boldsymbol{r}_{0}=\left(\begin{array}{c}
\frac{a}{2}-l \sin \phi \\
l \cos \phi \\
0
\end{array}\right)
$$

а вектор силы тяжести направляющей

$$
\overrightarrow{M g}=\left(\begin{array}{c}
0 \\
M g \\
0
\end{array}\right)
$$

то момент силы тяжести направляющей относительно точки $O_{1}$ будет:

$$
M_{O_{1}(\overrightarrow{M g})}=\boldsymbol{r}_{0} \cdot \overrightarrow{M g}=\left|\begin{array}{ccc}
\boldsymbol{i} & \boldsymbol{j} & \boldsymbol{k} \\
\frac{a}{2}-l \sin \phi & l \cos \phi & 0 \\
0 & M g & 0
\end{array}\right|=M g\left(\frac{a}{2}-l \sin \phi\right) \boldsymbol{k} .
$$

Момент реакции связи $T_{1}: M_{O_{1}\left(\boldsymbol{T}_{1}\right)}=\boldsymbol{r}_{A} \cdot \boldsymbol{T}_{1}=0$, так как $\boldsymbol{r}_{A} \| \boldsymbol{T}_{1}$ (рис. 4).

Момент силы $\boldsymbol{T}_{2}: M_{O_{1}\left(\boldsymbol{T}_{2}\right)}=\boldsymbol{r}_{B} \cdot \boldsymbol{T}_{2}$, где радиус-вектор определяется как

$$
\boldsymbol{r}_{B}=\left(\begin{array}{c}
a-l \sin \phi \\
l \cos \phi \\
0
\end{array}\right)
$$

вектор силы

$$
\boldsymbol{T}_{2}=\left(\begin{array}{c}
T_{2} \sin \phi \\
-T_{2} \cos \phi \\
0
\end{array}\right)
$$

тогда момент составит:

$$
M_{O_{1}\left(\boldsymbol{T}_{2}\right)}=\left|\begin{array}{ccc}
\boldsymbol{i} & \boldsymbol{j} & \boldsymbol{k} \\
a-l \sin \phi & l \cos \phi & 0 \\
T_{2} \sin \phi & -T_{2} \cos \phi & 0
\end{array}\right|=\boldsymbol{k} T_{2}[-\cos \phi(a-l \sin \phi)-\sin \phi \cdot l \cdot \cos \phi]=-a T_{2} \cos \phi \boldsymbol{k} .
$$


Сумма моментов сил, действующих на систему относительно точки $O_{1}$, будет:

$$
\begin{aligned}
\sum M\left(F_{i}\right)=m g(S-l \sin \phi) \boldsymbol{k}+M g\left(\frac{a}{2}-l \sin \phi\right) \boldsymbol{k}-a T_{2} \cos \phi \cdot \boldsymbol{k}= \\
=\left[m g(S-l \sin \phi)+M g\left(\frac{a}{2}-l \sin \phi\right)-a T_{2} \cos \phi\right] \boldsymbol{k} .
\end{aligned}
$$

Теорема об изменении кинетического момента запишется так:

$$
\begin{aligned}
\left\{m\left[(l-S \sin \phi) \ddot{\phi}-\ddot{S} \cos \phi-S \cos \phi(\dot{\phi})^{2}\right]+\right. & \left.M\left[\left(l-\frac{a}{2} \sin \phi\right) \ddot{\phi}-\frac{a}{2} \cos \phi(\dot{\phi})^{2}\right]\right\} l \cdot \boldsymbol{k}= \\
& =\left[m g(S-l \sin \phi)+M g\left(\frac{a}{2}-l \sin \phi\right)-a T_{2} \cos \phi\right] \boldsymbol{k} .
\end{aligned}
$$

Или, в проекции на вектор $\boldsymbol{k}$, составит:

$$
\begin{aligned}
\left\{m\left[(l-S \sin \phi) \ddot{\phi}-\ddot{S} \cos \phi-S \cos \phi(\dot{\phi})^{2}\right]+\right. & \left.M\left[\left(l-\frac{a}{2} \sin \phi\right) \ddot{\phi}-\frac{a}{2} \cos \phi(\dot{\phi})^{2}\right]\right\} l= \\
& =m g(S-l \sin \phi)+M g\left(\frac{a}{2}-l \sin \phi\right)-a T_{2} \cos \phi .
\end{aligned}
$$

Выразим из выражения (27) силу $T_{2}$ :

$$
\begin{aligned}
T_{2}=\frac{1}{a \cos \phi}\left\{m g(S-l \sin \phi)+M g\left(\frac{a}{2}-l \sin \phi\right)+\right. & l m\left[\ddot{S} \cos \phi+S \cos \phi \cdot(\dot{\phi})^{2}+(S \sin \phi-l) \ddot{\phi}\right]+ \\
& \left.+l M\left[\frac{a}{2} \cos \phi \cdot(\dot{\phi})^{2}+\left(\frac{a}{2} \sin \phi-l\right) \ddot{\phi}\right]\right\} .
\end{aligned}
$$

Далее запишем теорему о движении центра масс, имеем (см. [1]):

$$
\begin{aligned}
& (M+m) \ddot{x}_{c}=\left(T_{1}+T_{2}\right) \sin \phi, \\
& (M+m) \ddot{y}_{c}=(M+m) g-\left(T_{1}+T_{2}\right) \cos \phi,
\end{aligned}
$$

где $x_{c}, y_{c}$ - координаты центра масс системы: направляющая-поршень, м. При этом

$$
\begin{aligned}
& x_{c}=\frac{x_{n} \cdot m+x_{0} \cdot M}{m+M}=\frac{(S-l \sin \phi) m+\left(\frac{a}{2}-l \sin \phi\right) M}{m+M}=\frac{m S+M \frac{a}{2}}{m+M}-l \sin \phi, \\
& y_{c}=\frac{y_{n} \cdot m+y_{0} \cdot M}{m+M}=\frac{l \cos \phi \cdot m+l \cos \phi \cdot M}{M+m}=l \cos \phi .
\end{aligned}
$$

Дифференцируем выражение (31) и (32) дважды, получаем

$$
\begin{aligned}
& \ddot{x}_{c}=\frac{m \cdot \ddot{S}}{m+M}+l\left(\sin \phi(\dot{\phi})^{2}-\cos \phi \cdot(\ddot{\phi})\right), \\
& \ddot{y}_{c}=-l\left(\cos \phi \cdot(\dot{\phi})^{2}+\sin \phi \cdot(\ddot{\phi})\right) .
\end{aligned}
$$

Уравнения (29) и (30) с учетом выражений (33) и (34) примут вид:

$$
m \ddot{S}+l(M+m)\left(\sin \phi \cdot(\dot{\phi})^{2}-\cos \phi \cdot(\ddot{\phi})\right)=\left(T_{1}+T_{2}\right) \sin \phi .
$$

(34) примут вид:

$$
(M+m) l \cdot\left(\cos \phi \cdot(\dot{\phi})^{2}+\sin \phi \cdot(\ddot{\phi})\right)=\left(T_{1}+T_{2}\right) \cos \phi-(M+m) g .
$$

Преобразуем получившуюся систему уравнений (35) и (36) с целью исключения из системы сил $T_{1}$ и $T_{2}$. Для этого умножим уравнения (35) на $\cos \phi$, а $(36)$ на $(-\sin \phi)$ и, сложив их почленно, после преобразований получим:

$$
\ddot{\phi}+\frac{g}{l} \sin \phi=\frac{m \ddot{S} \cos \phi}{l(M+m)} .
$$

Имеем нелинейное уравнение вынужденных колебаний системы. Возбуждающей силой выступает движение поршня с массой $m$, значение которой определяется по формуле (12). Умножив 
уравнение (12) на $\cos \phi$ и подставив его в формулу (37), исключив неизвестное ускорение поршня $\ddot{S}$, имеем:

$$
\begin{aligned}
l \ddot{\phi}\left(M+m \sin ^{2} \phi+f m \sin \phi \cos \phi\right)+m l(\dot{\phi})^{2}(\sin \phi & \left.\cos \phi+f \cos ^{2} \phi\right)+ \\
& +(M+m) g \sin \phi+f m g \cos \phi=F(t) \cos \phi .
\end{aligned}
$$

Дифференциальное уравнение (38) - нелинейное второго порядка, его можно решить численно с начальными условиями $\phi(0)=0, \dot{\phi}(0)=0$. Затем с найденным значением $\phi(t)$ следует решать опять численно уравнение (12) или (37) с начальными условиями $S(0)=0, \dot{S}(0)=0$ и найти такой момент времени $t_{1}$, при котором $S\left(t_{1}\right)=a$. Затем сравнить полученную величину времени движения поршня с длительностью такта работы пульсатора. Уравнение (38) можно с допустимой погрешностью, пригодной для расчетов, упростить, чтобы получить аналитическое его решение. Если принять, что угол $\phi$ мал (рассматриваются малые колебания, что в нашем случае вполне уместно). Тогда $\sin \phi \approx \phi, \cos \phi \approx 1$. Пренебрегая малыми с $(M)$ величинами $m \phi^{2}$ и $f m \phi$ и отбрасывая нелинейное слагаемое, содержащее $(\dot{\phi})^{2}$, так как оно также мало при малых колебаниях, получаем упрощенное выражение колебания подвесной части доильного аппарата:

$$
M l \ddot{\phi}+(M+m) g \phi=F(t)-f m g .
$$

Решим уравнение (39) аналитически, заменим силу $F(t)$ его значением, после преобразований получим:

$$
\ddot{\phi}+\frac{(M+m) g \phi}{M l}=\frac{\left(P_{a}-P_{h}\right) S_{1}-f m g}{M l}-\frac{\left(P_{a}-P_{g}\right) S_{1} e^{-k t}}{M l} .
$$

Введем обозначения:

$$
\omega^{2}=\frac{(M+m) g}{M l}, \quad A=\frac{\left(P_{a}-P_{h}\right) S_{1}-f m g}{M l}(A>0), \quad B=\frac{\left(P_{a}-P_{g}\right) S_{1}}{M l}(B>0) .
$$

Тогда выражение (40) будет иметь вид

$$
\ddot{\phi}+\omega^{2} \phi=A-B e^{-k t} .
$$

Общее решение этого линейного неоднородного дифференциального уравнения имеет вид:

$$
\phi(t)=\phi_{\text {общ }}(t)+\phi_{\text {част }}(t) .
$$

Общее решение соответствующего однородного уравнения $\ddot{\phi}+\omega^{2} \phi=0$ будет:

$$
\phi_{\text {общ }}(t)=C_{1} \cos \omega t+C_{2} \sin \omega t .
$$

Частное решение будем искать в виде

$$
\phi_{\text {част }}(t)=C+D e^{-k t} .
$$

Дифференцируя выражение (44), получаем:

$$
\dot{\phi}_{\text {част }}(t)=(-k) D e^{-k t} ; \quad \ddot{\phi}_{\text {част }}(t)=(-k)^{2} D e^{-k t} .
$$

Подставляя предполагаемую форму частного решения (45) в (41), получаем:

$$
D\left(k^{2}+\omega^{2}\right) e^{-k t}+\omega^{2} C=A-B e^{-k t} .
$$

Приравнивая коэффициенты перед экспонентой и свободными членами, получаем систему:

$$
\left\{\begin{array}{l}
D\left(\omega^{2}+k^{2}\right)=-B, \\
C \cdot \omega^{2}=A .
\end{array}\right.
$$

Тем самым найдено частное решение уравнения (44):

$$
\phi_{\text {част }}(t)=\frac{A}{\omega^{2}}-\frac{B \cdot e^{-k t}}{\omega^{2}+k^{2}} .
$$

Тогда общее решение примет вид

$$
\phi(t)=C_{1} \cos \omega t+C_{2} \sin \omega t+\frac{A}{\omega^{2}}-\frac{B \cdot e^{-k t}}{\omega^{2}+k^{2}} .
$$




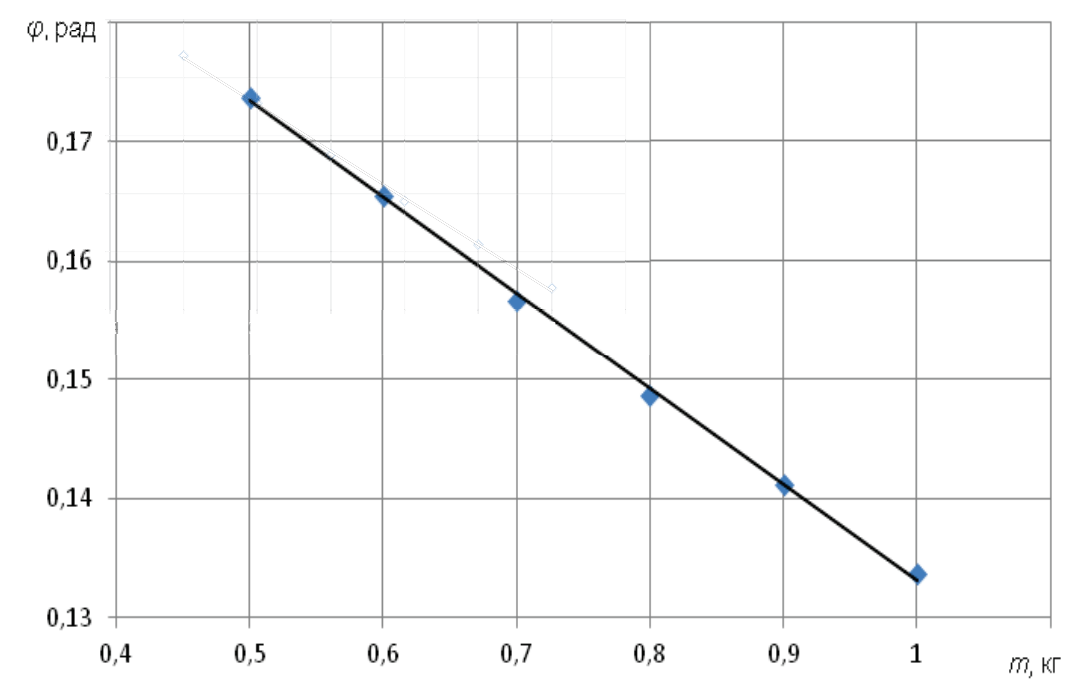

Рис. 5. Графическая зависимость угла отклонения подвеса от вертикального положения ( $\phi$, рад) от массы поршня $(m$, кг)

Для нахождении постоянных $C_{1}$ и $C_{2}$ уравнения (48) воспользуемся начальными условиями: $\phi(0)=0, \dot{\phi}(0)=0$. Имеем:

откуда

$$
0=C_{1} \cos 0+C_{2} \sin 0+\frac{A}{\omega^{2}}-\frac{B}{\omega^{2}+k^{2}},
$$

Далее

$$
C_{1}=\frac{B \omega^{2}-A\left(\omega^{2}+k^{2}\right)}{\omega^{2}\left(\omega^{2}+k^{2}\right)}
$$

$$
\dot{\phi}(t)=-C_{1} \omega \sin \omega t+C_{2} \omega \cos \omega t-\frac{B}{\omega^{2}+k^{2}}(-k) e^{-k t} .
$$

Подставляя в выражение (49) $t=0$, получаем:

откуда

$$
-C_{1} \omega \sin \omega 0+C_{2} \omega \cos \omega 0+\frac{B k}{\omega^{2}+k^{2}}=0,
$$

$$
C_{2}=-\frac{B k}{\omega\left(\omega^{2}+k^{2}\right)}
$$

$\mathrm{C}$ учетом найденных значений $C_{1}, C_{2}$ получаем решение уравнения (40) с начальными условиями $\phi(0)=0, \dot{\phi}(0)=0$ в виде:

$$
\phi(t)=\frac{B \omega^{2}-A\left(\omega^{2}+k^{2}\right)}{\omega^{2}\left(\omega^{2}+k^{2}\right)} \cos \omega t-\frac{B k}{\omega\left(\omega^{2}+k^{2}\right)} \sin \omega t+\frac{A}{\omega^{2}}-\frac{B e^{-k t}}{\left(\omega^{2}+k^{2}\right)} .
$$

Первые два слагаемых в формуле (50) описывают колебательный процесс с частотой $\omega$. Изменяя соотношение

$$
\omega^{2}=\frac{(M+m) g}{M l}
$$

можно добиться минимальной амплитуды колебания подвесной части доильного аппарата. Графические интерпретации выражения (50) представлены на рисунках 5 и 6.

Из рисунка 5 следует, что с увеличением массы поршня $m$ угол отклонения подвеса от вертикального положения $\phi$ уменьшается линейно. 


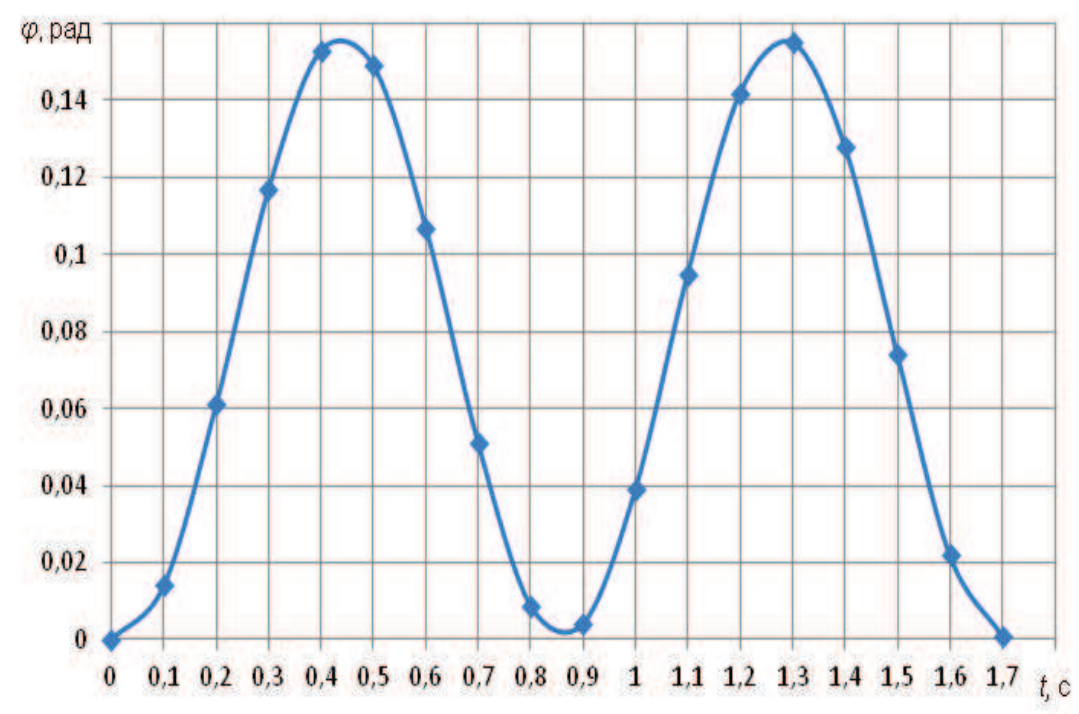

Рис. 6. Графическая зависимость угла отклонения подвеса от вертикального положения ( $\phi$, рад) от времени $(t, c)$ откачивания воздуха (работы системы) при массе поршня $m$ равной 0,8 кг

Из графика, представленного на рисунке 6 , видно, что рассматриваемая система совершает гармонические колебания. Для определения скорости и перемещения поршня в цилиндре коллектора решим совместно уравнения (12) и (38), исключая неизвестную $\ddot{\phi}$, получаем после преобразований:

$$
\begin{aligned}
& m\left(M+m \sin ^{2} \phi+f m \sin \phi \cos \phi\right) \ddot{S}= \\
& \quad+=(M+m)\left[(F(t)-f m g)-m g(\cos \phi-f \sin \phi) \sin \phi-m l(\dot{\phi})^{2}(\sin \phi+f \cos \phi)\right] .
\end{aligned}
$$

Замечание. Подставив в формулу (51) значение времени $t=0$ и воспользовавшись тем, что $\phi(0)=\dot{\phi}(0)=0$, получаем

$$
m M \ddot{S}(0)=(M+m)(F(0)-f m g),
$$

а так как $S(0)=\dot{S}(0)=0$, то необходимым условием начала движения поршня будет $\ddot{S} \geqslant 0$, что приводит к условию $F(0)-f m g \geqslant 0$.

Уравнение (51) не решается аналитически (см. [2]). Упростим его с достаточной погрешностью для инженерных расчетов, аналогично тому как было получено выражение (39). При малых углах $\phi$ из уравнения (51) получаем:

$$
\ddot{S}(t)=\frac{M+m}{m}\left(\frac{F(t)-f m g}{m}-g \phi(t)\right) .
$$

С учетом выражений (12) и (50) формула (52) примет вид:

$$
\ddot{S}(t)=\frac{M+m}{m}\left[\frac{\left(P_{a}-P_{h}\right) S_{1}\left(1-e^{-k t}\right)-f m g}{m}-g\left(C_{1} \cos \omega t+C_{2} \sin \omega t+\frac{A}{\omega^{2}}-\frac{B \cdot e^{-k t}}{\left(\omega^{2}+k^{2}\right)}\right)\right] .
$$

Но согласно принятых ранее обозначений значения коэффициентов $A$ и $B$ определяются как:

$$
A=\frac{\left(P_{a}-P_{h}\right) S_{1}-f m g}{M l}, \quad B=\frac{\left(P_{a}-P_{h}\right) S_{1}}{M l},
$$


тогда выражение (53) с учетом их значений будет

$$
\ddot{S}(t)=(M+m)\left[\frac{l}{m}\left(A-B e^{-k t}\right)-\frac{g}{M}\left(C_{1} \cos \omega t+C_{2} \sin \omega t+\frac{A}{\omega^{2}}-\frac{B \cdot e^{-k t}}{\left(\omega^{2}+k^{2}\right)}\right)\right] .
$$

Интегрируя выражение (54), определяем скорость поршня:

$$
\begin{aligned}
& \dot{S}(t)=\dot{S}(0)+\int_{0}^{t} \ddot{S}(t) d t= \\
& =(M+m)\left[\frac{l}{M}\left(A t-\left.\frac{B e^{-k t}}{(-k)}\right|_{0} ^{t}\right)-\frac{g}{M}\left(\left.\frac{C_{1}}{\omega} \sin \omega t\right|_{0} ^{t}-\left.\frac{C_{2}}{\omega} \cos \omega t\right|_{0} ^{t}+\frac{A t}{\omega^{2}}-\left.\frac{B \cdot e^{-k t}}{\left(\omega^{2}+k^{2}\right)(-k)}\right|_{0} ^{t}\right)\right]= \\
& =(M+m)\left[\frac{l}{M}\left(A t+\frac{B e^{-k t}}{k}-\frac{B}{k}\right)-\right. \\
& \left.\quad-\frac{g}{M}\left(\frac{C_{1}}{\omega} \sin \omega t-\frac{C_{2}}{\omega} \cos \omega t+\frac{C_{2}}{\omega}+\frac{A t}{\omega^{2}}+\frac{B \cdot e^{-k t}}{\left(\omega^{2}+k^{2}\right) k}-\frac{B}{\left(\omega^{2}+k^{2}\right)}\right)\right] .
\end{aligned}
$$

Интегрируя уравнение (55), получаем закон движения поршня:

$$
\begin{aligned}
S(t)=S(0)+ & \int_{0}^{t} \dot{S}(t) d t=(M+m)\left[\frac{l}{M}\left(\frac{A t^{2}}{2}+\left.\frac{B e^{-k t}}{k(-k)}\right|_{0} ^{t}-\frac{B l t}{k}\right)-\right. \\
& \left.\quad-\frac{g}{M}\left(-\left.\frac{C_{1}}{\omega^{2}} \cos \omega t\right|_{0} ^{t}-\left.\frac{C_{2}}{\omega^{2}} \sin \omega t\right|_{0} ^{t}+\frac{C_{2} t}{\omega}+\frac{A t^{2}}{2 \omega^{2}}+\left.\frac{B \cdot e^{-k t}}{k\left(\omega^{2}+k^{2}\right)(-k)}\right|_{0} ^{t}-\frac{B t}{k\left(\omega^{2}+k^{2}\right)}\right)\right] \\
S(t)= & (M+m)\left[\frac{A l t^{2}}{2 m}-\frac{B l\left(e^{-k t}-1\right)}{m k^{2}}-\frac{B l t}{m k}-\right. \\
& \left.\quad-\frac{g}{M}\left(\frac{C_{1}(1-\cos \omega t)}{\omega^{2}}-\frac{C_{2}}{\omega^{2}} \sin \omega t+\frac{C_{2} t}{\omega}+\frac{A t^{2}}{2 \omega^{2}}-\frac{B \cdot\left(e^{-k t}-1\right)}{k^{2}\left(\omega^{2}+k^{2}\right)}-\frac{B t}{k\left(\omega^{2}+k^{2}\right)}\right)\right],
\end{aligned}
$$

где постоянные члены, входящие в выражение, определяются по формулам:

$$
C_{1}=\frac{B \omega^{2}-A\left(\omega^{2}+k^{2}\right)}{\omega^{2}\left(\omega^{2}+k^{2}\right)}, \quad C_{2}=-\frac{B k}{\omega\left(\omega^{2}+k^{2}\right)}, \quad A=\frac{\left(P_{a}-P_{h}\right) S_{1}-f m g}{M l}, \quad \omega=\sqrt{\frac{(M+m) g}{M l}} .
$$

При достижении поршнем торцовой стенки цилиндра происходит его удар о стенку. Угол отклонения тяг подвесов от вертикали при этом будет $\phi_{0}$. Так как на поршень действует перепад давлений (вакуум), то при ударе отскока поршня от стенки не произойдет, т. е. удар можно считать неупругим. До удара поршень и направляющая двигались противоположно друг другу. После удара относительное движение поршня отсутствует, поршень с направляющей будут двигаться совместно с одной скоростью. В это время пульсатор должен переключиться для изменения тактов и перепада давлений, действующего на поршень (см. [9]). Далее закон изменения колебаний системы будет происходить аналогично рассмотренному выше, но при движении в противоположном направлении.

3. Результаты и выводы. Получено нелинейное уравнение вынужденных колебаний системы на гибких подвесах. Где возбуждающей силой выступает движение поршня, которое изменяет центр масс системы. Дифференциальное уравнение, нелинейное второго порядка, можно решить численно с начальными условиями $\phi(0)=\dot{\phi}(0)=0$. Затем с найденным значением $\phi(t)$ следует решать опять численно уравнение (12) или (37) с начальными условиями $S(0)=0, \dot{S}(0)=0$ и найти такой момент времени $t_{1}$, при котором $S\left(t_{1}\right)=a$. Затем соизмерить полученную величину времени движения поршня с длительностью такта работы пульсатора. С достаточной погрешностью для инженерных расчетов можно применять представленные приближенные аналитические решения дифференциальных уравнений вынужденных колебаний системы на гибких подвесах. 


\section{СПИСОК ЛИТЕРАТУРЫ}

1. Жуковский Н. Е. Теоретическая механика. - М.: ГИТТЛ, 1952.

2. Лаврентъев М. А. Методы теории функций комплексного переменного. - М.: Лань, 2002.

3. Никитин E. М. Краткий курс теоретической механики. - М.: Наука, 1971.

4. Талалаев Г. Д. Теория и расчет доильных аппаратов. - Вологда, 1990.

5. Улъянов В. М. Вопросы теории машинного доения. - Рязань, 2006.

6. Ульянов В. М., Киръянов А. Ю., Панферов Н. С., Набатчиков А. В. Высокоэффективный доильный аппарат// в кн.: Сб. мат. Всеросс. науч.-практ. круглого стола. - Рязань: Академия ФСИН России, 2017.

7. Ульянов B. М., Хрипин В. А. Физиологически адаптированный доильный аппарат// Сельский механизатор. - 2007. - 1. - С. 34.

8. Ульянов B. М., Хрипин В. А. Патент Российской Федерации. Доильный аппарат/ Опубл. 20.05.2007.

9. Ульянов В. М., Хрипин В. А., Карпов Ю. Н., Набатчиков А. В. Теоретические исследования доильного аппарата с изменяющимся центром масс// Вестн. Рязан. гос. агротехн. ун-та им. П. А. Костычева. 2014. - 4. - C. 81-86.

Ульянов Вячеслав Михайлович

Рязанский государственный агротехнологический университет им. П. А. Костычева

E-mail: ulyanov-v@list.ru

Хрипин Владимир Александрович

Академия права и управления Федеральной службы исполнения наказаний России, Рязань

E-mail: khripin@mail.ru

Кирьянов Александр Юрьевич

Академия права и управления Федеральной службы исполнения наказаний России, Рязань

E-mail: customs.rzn.kay@yandex.ru 